\title{
Intención emprendedora de los estudiantes universitarios
}

\author{
Entrepreneurial intention of university students \\ Angélica Beatriz Contreras Cueva \\ Olga González Morales \\ Pamela Macías Álvarez
}

\begin{abstract}
RESUMEN
En este estudio se analizan las actitudes de los estudiantes universitarios que configuran su intención emprendedora mediante la Teoría de Acción Razonada, identificando posibles diferencias entre dos centros universitarios utilizados como casos de estudio. El objetivo, por una parte, es analizar la influencia de la actitud, los aspectos de control y el factor social de los estudiantes sobre su intención emprendedora, y por otra, las ventajas y obstáculos al del emprendimiento sobre la actitud del alumno. Para el análisis se aplicaron el modelo de ecuaciones estructurales y la prueba $T$ para diferencia de medias a una muestra de 1117 estudiantes. Los resultados apuntan a que existen diferencias entre los dos centros universitarios en cuanto a la opinión de los factores analizados, sin embargo, ambos tienen intención emprendedora. Por otra parte se evidencia que la intención emprendedora está influenciada por la actitud de los estudiantes y el control de la conducta, además de que las ventajas y los obstáculos impactan sobre la actitud de emprender al igual que la norma subjetiva.

Palabras clave: actitudes emprendedoras, Teoría de la Acción Razonada, Teoría del Comportamiento, estudiantes universitarios, ecuaciones estructurales.
\end{abstract}

\begin{abstract}
In this study, the attitudes of university students that shape their entrepreneurial intention are analyzed through the Theory of Reasoned Action, identifying possible differences between two Mexican university centers used as case studies. The objective, from one point of view, is to analyze the influence of students' attitude, control features and social factor on their entrepreneurial intention; and from another one, the advantages and obstacles of entrepreneurship over the student's attitude. For the analysis, the structural equation model and the $\mathrm{T}$ test for mean difference were applied to a sample of 1117 students. The results indicate that there are differences between the two university centers, regarding the opinion of the factors analyzed; however, both have entrepreneurial intent. On the other hand, it is evident that the entrepreneurial intention is influenced by the students' attitude and the behavior control, in addition to the fact that the advantages and obstacles impact on the entrepreneurial attitude as well as the subjective norm.

Keywords: entrepreneurial attitudes, Theory of Reasoned Action, Theory of Behavior, university students, structural equations.
\end{abstract}




\section{INTRODUCCIÓN}

El concepto de empresario ha ido evolucionando a lo largo de los años, poniendo especial énfasis en el aspecto innovador como motor de cambio y generador de iniciativas empresariales diferentes a las tradicionales. Autores como Acs, Audretsch, Braunerhjelm y Carlsson (2003) o Audretsch, Keilbach y Lehmann (2005) han desarrollado su investigación en el empresario como agente clave en la transformación de los nuevos conocimientos en mejores productos, procesos, organizaciones o accesos a nuevos mercados. Este planteamiento tiene sus raíces en el empresario innovador, desarrollado por Schumpeter (1950), que lo considera factor esencial en el desarrollo económico. Asimismo, los estudios del Global Entrepreneurship Monitor (GEM) consideran que la intención de crear negocios, empresas, o la expansión de una existente, incluyendo el autoempleo, es iniciativa empresarial, reconocen esta como la determinante del crecimiento económico (Reynolds, Hay y Camp, 1999).

Es innegable el papel que la creación de empresas tiene sobre los objetivos de crecimiento y pleno empleo (Ackermann y Audretsch, 2013; Thurik, Stam y Audretsch, 2013). Por ello, las líneas de actuación pública convierten en una prioridad el fomento de la iniciativa empresarial y de sus actitudes asociadas, sobre todo, insisten en incluir acciones y medidas que logren objetivos y competencias clave en los diferentes niveles educativos. Tanto los gobiernos como las instituciones necesitan tener conocimiento de las características de los potenciales emprendedores y de su entorno para la toma de decisiones que derive en una planificación coherente y efectiva. La universidad no está al margen de este proceso, dado que una parte importante de las

Angélica Beatriz Contreras Cueva. Profesora-investigadora adscrita al Departamento de Métodos Cuantitativos de la Universidad de Guadalajara, México. Es doctora en empresas y cuenta con especialidad Técnicas y análisis de la Empresa. Entre sus publicaciones recientes se encuentran "Oportunidades de los graduados universitarios en el mercado laboral mexicano: diferencias por sexo y formación” (2020) y "La influencia de la educación y otras variables relevantes en la decisión de emprender: dos casos de estudio comparados de estudiantes universitarios de Ciencias Sociales y Jurídicas de España y México” (2019). Su línea de investigación es educación y empleo, y emprendimiento. Correo electrónico: acontre@cucea.udg.mx. ID: https://orcid. org/0000-0002-3057-1272.

Olga González Morales. Profesora del Departamento de Economía Aplicada y Métodos Cuantitativos de la Universidad de La Laguna, Canarias, España. Es miembro del Instituto Universitario de Investigación Social y Turismo (ISTUR) y Coordinadora del Grupo de Investigación Políticas Publicas y Desarrollo Regional (PpyDER). Ha participado en numerosos proyectos nacionales e internacionales. Correo electrónico: olgonzal@ull.edu.es. ID: https://orcid.org/0000-0002-3754-2300.

Pamela Macías Álvarez. Profesora de Asignatura B adscrita al Departamento de Métodos Cuantitativos de la Universidad de Guadalajara, México. Se ha desempeñado como asesora organizacional orientada a modelos de calidad y optimización de costos e inventario. Entre sus publicaciones recientes se encuentran "Incidencia de la certificación Tesoros de México sobre la ventaja competitiva de los hoteles" (2018) y "Análisis de las políticas de emprendimiento en la Educación Superior en México. Investigaciones de economía de la educación” (2019). Correo electrónico: pamela.macias@cucea.udg.mx. ID: https://orcid. org/0000-0002-6920-447X. 
acciones dirigidas a mejorar la empleabilidad de sus estudiantes es potenciar actitudes emprendedoras que puedan influir en futuras intenciones emprendedoras.

Existen diversos factores que influyen en la decisión de emprender ligados a un conjunto de motivos y obstáculos, entre los cuales ha primado como obstáculo relevante los factores económicos. Recientemente, las investigaciones se dirigen hacia el estudio de las intenciones emprendedoras más que a los atributos y características personales (Echeverri, Valencia, Benjumea y Barrera, 2018; Valencia, Restrepo y Restrepo, 2015).

Este estudio parte del supuesto de identificar posibles diferencias entre los estudiantes de dos centros universitarios, el CUCEA, que imparte licenciaturas en torno a las ciencias económico-administrativas, y el CUCEI, que imparte licenciaturas en ciencias exactas, bajo el supuesto de que, por un lado, los alumnos de negocios tienden a tener mayores intenciones de convertirse en empresarios, dado que tienen mejor formación para ello, y por otro lado los estudiantes de ciencias exactas tienden a generar en mayor medida innovaciones, lo que conlleva una formación transversal que promueva el emprendimiento (Gutiérrez, Asprilla y Gutiérrez, 2014), lo que permite fundamentar la relevancia de esta comparación sobre la contribución de cada área de formación al emprendimiento.

El objetivo de este trabajo es analizar, mediante la Teoría de Acción Razonada (TAR), la influencia que tiene la percepción de los estudiantes universitarios sobre su intención emprendedora, tomando en consideración los siguientes aspectos: la actitud ante el emprendimiento, el control de la conducta, el factor social, las ventajas y los obstáculos para emprender. Conocer estas relaciones podría ser de utilidad para evaluar la pertinencia de los programas educativos de acuerdo a la misión y visión de los centros universitarios y apoyará a las autoridades para la instrumentación de políticas públicas que coadyuven al fortalecimiento de la universidad emprendedora.

En los siguientes apartados se desarrolla el marco teórico del estudio, se continúa con la metodología y las técnicas utilizadas para el análisis de los datos, así como se describen las variables utilizadas. En el cuarto apartado se desarrollan los resultados, para finalmente terminar con la discusión y las conclusiones en los apartados quinto y sexto.

\section{Marco TEÓRICo y REVISIÓN DE LITERATURA}

El modelo de comportamiento humano que se toma como referencia para este estudio es el de la Teoría de la Acción Razonada (TAR) propuesta por Ajzen y Fishbein $(1974,1980)$. Dicha teoría sostiene que la actitud de las personas, en esencia racionales, les permite ejercer acciones a partir de la información, cuando evalúan estas como positivas y creen que las personas que consideran socialmente relevantes lo aprueban. Esto explica que la intención esté en función de la actitud hacia la conducta y los 
aspectos sociales o norma subjetiva. Las actitudes hacia la conducta son las creencias que dirigen el comportamiento y pertenecen a los individuos. Los aspectos sociales o normas subjetivas son la influencia que ejercen los individuos o grupos sociales que son importantes para el que realiza la acción.

En general, la literatura demuestra que las investigaciones sobre iniciativa empresarial se han abordado desde distintos enfoques con modelos centrados en rasgos de personalidad (Brockhaus, 1982; McClelland, 1961), de conducta y de intenciones (Gartner, 1985; Van de Ven, Hudson y Schroeder, 1984), y, posteriormente, otros que incorporan los antecedentes de las actitudes para estimar el proceso del emprendedor (Boyd y Vozikis, 1994; Krueger, Reilly y Carsrud, 2000; Shapero, 1975), demostrando que la actitud es determinante en la acción (González, Estrada, Leite y Álvarez, 2017).

Respecto a las investigaciones sobre las actitudes hacia la creación de empresas, los resultados de los estudios de Becker (1976), Hirshleifer (1977), López y Serrano (2017), Rubin y Paul (1979) y Skaperdas (1992) convergen al considerar la determinación endógena de las preferencias y de la actitud hacia el riesgo. También Barsky, Juster, Kimball y Shapiro (1997), Robson (1996) y Waldman (1994) se acercan a la idea de que la aversión al riesgo puede cambiar con el transcurso del tiempo, aumentando con la edad y los ingresos.

Un factor analizado con recurrencia es el nivel de estudios de los emprendedores. Los análisis de Brunello (2002) pusieron en evidencia que un mayor nivel educativo se asociaba a un menor espíritu emprendedor, a pesar de que podría ayudar a afrontar los problemas que van surgiendo en el desarrollo de la actividad, tal y como señalan Cooper, Woo y Gimeno (1994). No obstante, determinados tipos de estudios, como los relacionados con el campo de la economía y la administración de empresas, sí ayudan especialmente en el proceso emprendedor (Gasse, 1985; Hisrich, 1990). Los cursos formativos son otra opción alternativa o complementaria para los emprendedores. Nabi, Liñan, Krueger, Fayolle y Walmsley (2016) y Ritsila y Tervo (2002) indican que las personas que participan en mayor medida en cursos de formación tienen más probabilidad de convertirse en empresarios independientes. En este sentido, Guerrero, Cunningham y Urbano (2015) destacan la contribución de la educación emprendedora al desarrollo económico y social dada la importancia del capital de conocimiento como fuente de ventaja competitiva.

Sin embargo, estudios más recientes concluyen que la educación superior aumenta la probabilidad de establecerse por cuenta propia, así como los ingresos, especialmente en el caso de las mujeres empresarias (Atherton, Faria, Wu y Wu, 2015), con lo cual las universidades deberían replantearse su papel en la mejora de competencias clave, para el individuo y para el mercado laboral, desde dos ópticas complementarias: el papel de las universidades en los sistemas de innovación (Breznitz y Etzkowitz, 2016; Godin y Gingras, 2000; Sampat, Mowery y Ziedonis, 2003) y el papel de las universidades 
en el fomento de la empleabilidad y el emprendimiento de sus estudiantes (Blázquez, Zaldívar y Leite, 2018; Clark, 1998a y 1998b; Etzkowitz y Leydesdorff, 2000; Kirby, 2006). Según Etzkowitz (2003), la Universidad ha pasado de su primera función, la docencia, a la inclusión y el engarce entre la docencia y la investigación, que permite a sus estudiantes aprender nuevas competencias útiles en el mercado laboral y reducir la incertidumbre (Arroyo, 2016; González et al., 2017; Xerfan, Ribeiro y Nunes, 2018).

Otras investigaciones han abordado estudios en el ámbito del emprendimiento y la TAR, en concreto, Rueda, Fernández y Herrero (2013) analizan el efecto de las ventajas y los inconvenientes percibidos en el emprendimiento sobre la intención de crear un negocio propio, y Salem (2014) determina en qué medida las percepciones y las habilidades de los estudiantes afectan en su intención emprendedora y su efecto al tomar una materia relacionada con el emprendimiento.

Por su parte, Marulanda, Correa y Mejía (2009) sostienen que para que una persona desarrolle enfoques emprendedores debe poseer factores internos de personalidad (motivación hacia el logro, independencia, creatividad) y factores culturales, familiares, laborales que interactúen para potencializar las habilidades emprendedoras. Posteriormente, Marulanda, Montoya y Vélez (2014) integran en el análisis los incentivos que el gobierno y otros organismos crean para fomentar el emprendimiento y concluyen que más allá de esto, el proceso de creación de empresas se determina por la percepción del individuo, tanto a nivel interno (en relación con sus objetivos y capacidades) como de la viabilidad externa, la cual depende de la imagen de la actividad a nivel social y del apoyo que recibe de las personas que considera más importantes en su vida.

\section{Metodología}

Partiendo de estas premisas, en este apartado se presentan la metodología y las técnicas utilizadas para el análisis de los datos, así como las descripciones de las variables utilizadas. El estudio que se realiza es de naturaleza cuantitativa aplicado empíricamente para contrastar las hipótesis encaminadas a validar las actitudes hacia la intención de los estudiantes de la Universidad de Guadalajara.

Para la obtención de los datos se utilizó parte de las preguntas aplicadas previamente en el cuestionario diseñado para el proyecto de investigación que se está llevando a cabo entre la Universidad de La Laguna (ULL) de España y el Centro Universitario de Ciencias Económico-Administrativas (UdeG) de México (Anexo), y fue ajustado con las preguntas de otros proyectos sobre emprendimiento (Liñán y Chen, 2009; Rueda, Fernández y Herrero, 2013).

La población objeto de estudio fueron los estudiantes matriculados en los centros universitarios de Ciencias Económico-Administrativas (CUCEA), curso 2014-2015, y Ciencias Exactas (CUCEI), curso 2015-2016, de la Universidad de Guadalajara (UDG). 
Del repaso de la literatura y del modelo establecido se establecen las siguientes hipótesis:

$\mathrm{H}_{1}$. La intención emprendedora está influenciada positivamente por la actitud de los estudiantes.

$\mathrm{H}_{2}$. La intención emprendedora esta influenciada por el control de la conducta del estudiante universitario.

$\mathrm{H}_{3}$. La intención emprendedora está impactada positivamente por la norma subjetiva que los estudiantes tienen.

$\mathrm{H}_{4}$. La actitud emprendedora del estudiante universitario está influenciada positivamente por las ventajas para emprender.

$\mathrm{H}_{5}$. La actitud emprendedora del estudiante universitario está influenciada negativamente por los obstáculos para emprender.

$\mathrm{H}_{6}$. Existen diferencias entre los estudiantes de ciencias exactas y económicoadministrativas.

Para el análisis de la información se seleccionaron del cuestionario (ver Anexo) los apartados sobre la intención emprendedora y la percepción para emprender. Las valoraciones se capturaron a través de escalas Likert de cinco posiciones, donde 1 significa totalmente en desacuerdo y 5 totalmente de acuerdo.

Para contrastar el modelo establecido se utilizó el modelo de ecuaciones estructurales (SEM), el cual combina la regresión múltiple con el análisis factorial confirmatorio, es útil para explicar la causalidad entre las diferentes variables latentes, las cuales se miden a través de indicadores (Diamantopoulos, 1994). Las ventajas de usar SEM son, por una parte, la incorporación de variables latentes, que solo pueden ser medidas a través de la utilización de variables observables y, por otra, permite integrar simultáneamente varias ecuaciones de regresión múltiple, que pueden estimar los efectos indirectos, generados por variables mediadoras, y los efectos totales, definidos como la suma de los efectos directos e indirectos (Barrio y Luque, 2000).

Un aspecto fundamental del SEM es ser una herramienta para evaluar los modelos teóricos (en este estudio es la TAR) que involucran relaciones causales sobre datos no experimentales cuando las relaciones son de tipo lineal (Kerlinger y Lee, 2002). Los SEM realmente no prueban la causalidad, sin embargo, ayudan a seleccionar las hipótesis causales relevantes, eliminando las que no son sustentadas por la evidencia empírica.

En los modelos SEM, el análisis factorial confirmatorio (AFC) valora las propiedades métricas de las escalas de medida, y para esta valoración, la teoría, más que los datos numéricos, es la que se utiliza para la evaluación.

El método para la estimación de los parámetros y el proceso de modelización se realiza en dos etapas: el submodelo de medida y el submodelo estructural. El submodelo de medida establece la relación de las variables latentes con las observables (Bollen, 1989), mostrando la fiabilidad y la validez de los indicadores como medidas de las variables 
latentes. El submodelo estructural establece cómo las variables latentes se relacionan entre sí (Bollen, 1989), confirman la relación y consistencia del modelo con los datos.

El SEM propone seis pasos para su aplicación:

I. La especificación del modelo. Es el planteamiento de la teoría, que sustenta el estudio con las ecuaciones, establece los efectos causales de las variables latentes y su relación con las variables observables.

II. Identificación del modelo. Para la identificación del modelo se debe de asegurar que los parámetros puedan ser estimados, al verificar que se dispone de una expresión algebraica para cada uno, que se exprese en función de las varianzas y covarianzas muestrales.

III. Evaluación de la calidad de la base de datos. Previo al análisis se debe evaluar la calidad de la base de datos para que el tamaño de la muestra sea el adecuado. Jackson (2003) recomienda para el SEM una muestra mínima de 200 sujetos. Otro aspecto a considerar es revisar la multicolinealidad de las variables; para revisar la existencia de esta se recomienda verificar que $\mathrm{r}<0,85$ mediante correlación bivariada (Kline, 2005). Finalmente, se debe considerar que los estadísticos utilizados en el SEM tienen distribución normal.

IV. Estimación de los parámetros. La estimación implica determinar los valores de los parámetros desconocidos. La técnica para la estimación es la de máxima verosimilitud (MV), la cual cumple los supuestos de normalidad multivariada. La sensibilidad a la no normalidad del modelo genera la necesidad de utilizar técnicas alternativas como las de mínimos cuadrados ponderados (WLS), generalizados (GLS) y, para casos de muestras mayores o iguales a 500, la técnica asintóticamente libre de distribución (AGL).

V. Evaluación del ajuste e interpretación. Esta etapa es útil para conocer si el modelo es correcto, precisando su poder de predicción y sirviendo como aproximación del fenómeno real. Para evaluar el ajuste del modelo existen múltiples indicadores, como el estadístico Chi-cuadrado, el índice de ajuste comparativo (CFI), el error cuadrático medio de aproximación (RMSEA), entre otros. Chi-cuadrado debe ser no-significativo, lo que representa un buen ajuste de los datos, si el estadístico fuera significativo demostraría que la estructura del modelo teórico propuesto es significativamente diferente de la indicada por la matriz de covarianza de los datos, sin embargo, debe recordarse que el Chi cuadrado es sensible al tamaño de la muestra. CFI varía, por lo general, entre 0 y 1 , valores superiores a 0.9 y 0.95 sugieren un ajuste satisfactorio y óptimo entre las estructuras teóricas y los datos empíricos. El índice RMSEA se considera óptimo cuando sus valores son menores a 0.06. 
Finalmente debe considerarse la significación de los parámetros estimados, además del ajuste del modelo.

VI. Reespecificación del modelo. Este procedimiento permite determinar si el modelo obtenido es el más adecuado, en caso de ser necesario, con los métodos previos se puede seleccionar parámetros a añadir o eliminar del modelo original, cambios que deberán fundamentarse en la teoría; con esta acción se esperaría una mejora en los índices de bondad de ajuste. La reespecificación se analiza a partir de los índices de modificación, que corresponde a la reducción del Chi-cuadrado. El valor superior de 3.84 sugiere una reducción estadísticamente significativa.

Tabla 1. Descripción de los estudiantes de las muestras.

\begin{tabular}{|c|c|c|c|c|c|}
\hline & \multirow[b]{2}{*}{ Variable } & \multicolumn{2}{|c|}{ CUCEA } & \multicolumn{2}{|c|}{ CUCEI } \\
\hline & & Hombres & Mujeres & Hombres & Mujeres \\
\hline Sexo & & 47.27 & 52.73 & 84.97 & 15.03 \\
\hline Edad & Promedio mediano & 22 & 21 & 22 & 22 \\
\hline \multirow{3}{*}{$\begin{array}{l}\text { Tipo de institución } \\
\text { de los estudios } \\
\text { previos a la } \\
\text { Universidad }\end{array}$} & Ambos & 0.67 & 0.13 & & \\
\hline & Privado & 9.45 & 8.39 & 10.11 & 1.37 \\
\hline & Público & 37.15 & 44.21 & 74.86 & 13.66 \\
\hline \multirow[t]{13}{*}{ Carrera } & Administración & 6.79 & 7.72 & & \\
\hline & Administración Financiera y Sistemas & 3.20 & 2.40 & & \\
\hline & Administración Gubernamental y Políticas Públicas & 2.26 & 1.60 & & \\
\hline & Economía & 2.93 & 0.93 & & \\
\hline & Gestión y Economía Ambiental & 1.73 & 2.00 & & \\
\hline & Contaduría Pública & 8.52 & 7.59 & & \\
\hline & Mercadotecnia & 6.13 & 6.66 & & \\
\hline & Negocios Internacionales & 6.66 & 7.72 & & \\
\hline & Recursos Humanos & 2.26 & 5.06 & & \\
\hline & Sistemas de Información & 1.60 & 0.40 & & \\
\hline & Turismo & 5.19 & 10.65 & & \\
\hline & Informática & & & 27.32 & 6.28 \\
\hline & Ingeniería en Computación & & & 57.65 & 8.74 \\
\hline
\end{tabular}

Fuente: Elaboración propia.

Por último, para identificar las posibles diferencias de los estudiantes ante la intención emprendedora de los centros universitarios analizados, se utilizó la prueba $\mathrm{T}$ de Student para dos muestras independientes. Esta prueba permite determinar la 
igualdad entre dos grupos independientes; en este caso determina la significación entre los estudiantes de ciencias exactas y ciencias económico-administrativas. Se acepta la hipótesis de igualdad siempre que p-value sea mayor a 0.05.

En cuanto a los datos, se seleccionaron muestras estratificadas por cada carrera al 95\% de nivel de confianza y 5\% de error muestral. Se obtuvo una muestra de 751 estudiantes de las carreras del CUCEA-UDG y 366 de las carreras del CUCEI-UDG, matriculados en 2014 y 2015. La tabla 1 resume los estudiantes por cada carrera y por cada centro.

\section{Resultados}

Esta sección recoge el análisis del submodelo de medida, mediante la validación de las escalas utilizadas para cada una de las variables incluidas en el modelo y el análisis del submodelo estructural. Con fines comparativos se realiza una prueba de diferencia de medias entre centros universitarios. Con los parámetros y criterios arrojados del modelo de ecuaciones estructurales se realiza la interpretación de los resultados de acuerdo con los pasos descritos con anterioridad en la sección de metodología.

La especificación del modelo (paso I) se sustenta en la Teoría de la Acción Razonada (TAR), tal y como se comentó anteriormente. Esta teoría de la conducta humana considera a la intención como un indicador de la conducta. Las variables que explican la intención son la actitud hacia el comportamiento y el factor social o norma subjetiva (Ajzen y Fishbein, 1980).

Figura 1. Teoría de la Acción Razonada.

Creencias de que la conducta conlleva consecuencias y resultados

Creencias del individuo sobre sí mismo, de cómo se percibe ante la facilidad o dificultad de realizar la acción

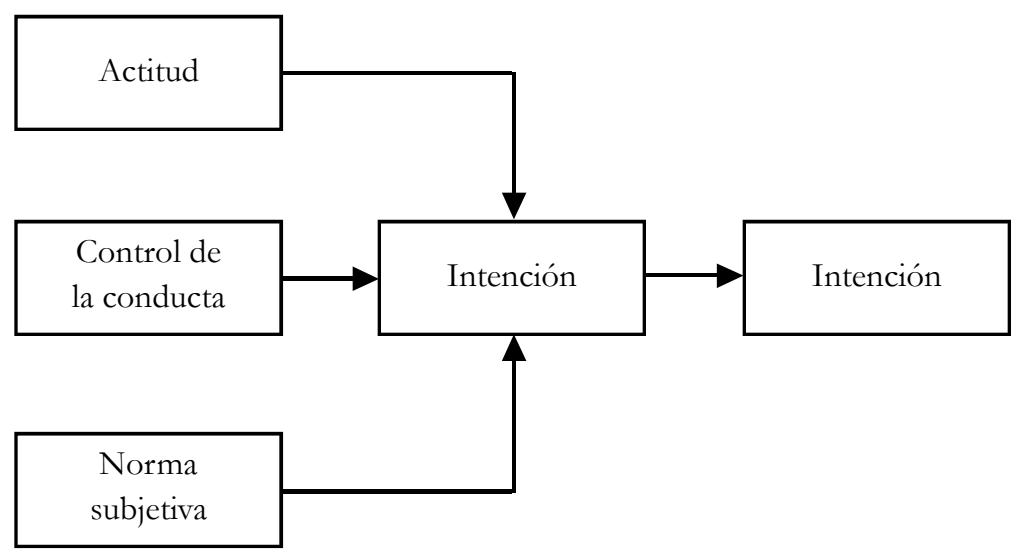

Creencias acerca de si determinadas personas o grupos específicos consideran que debería realizar o no un comportamiento

Fuente: Liñan y Chen, 2009.

La figura 1 presenta el esquema de la TAR y muestra los constructos que intervienen. Se observa que, detrás de la conducta del individuo, están sus creencias. 
La especificación del modelo incluirá tres variables explicativas que dan sentido a la intención para crear empresas: la actitud, el factor social y el control (Shapero y Sokol, 1982).

La actitud está condicionada por las creencias que el estudiante tiene hacia el emprendimiento, considerándolas como ventajas o no (obstáculos).

El factor social o norma subjetiva se refiere a la presión social que el futuro empresario percibe de las personas de referencia y que influyen sobre su conducta.

La variable control conductual está definida como la percepción que el individuo tiene sobre la facilidad o no de convertirse en empresario.

La especificación del modelo estructural se presenta en la figura 2. Los significados de las variables y el contenido del cuestionario se apuntan en el Anexo.

Figura 2. Especificación del modelo estructural.

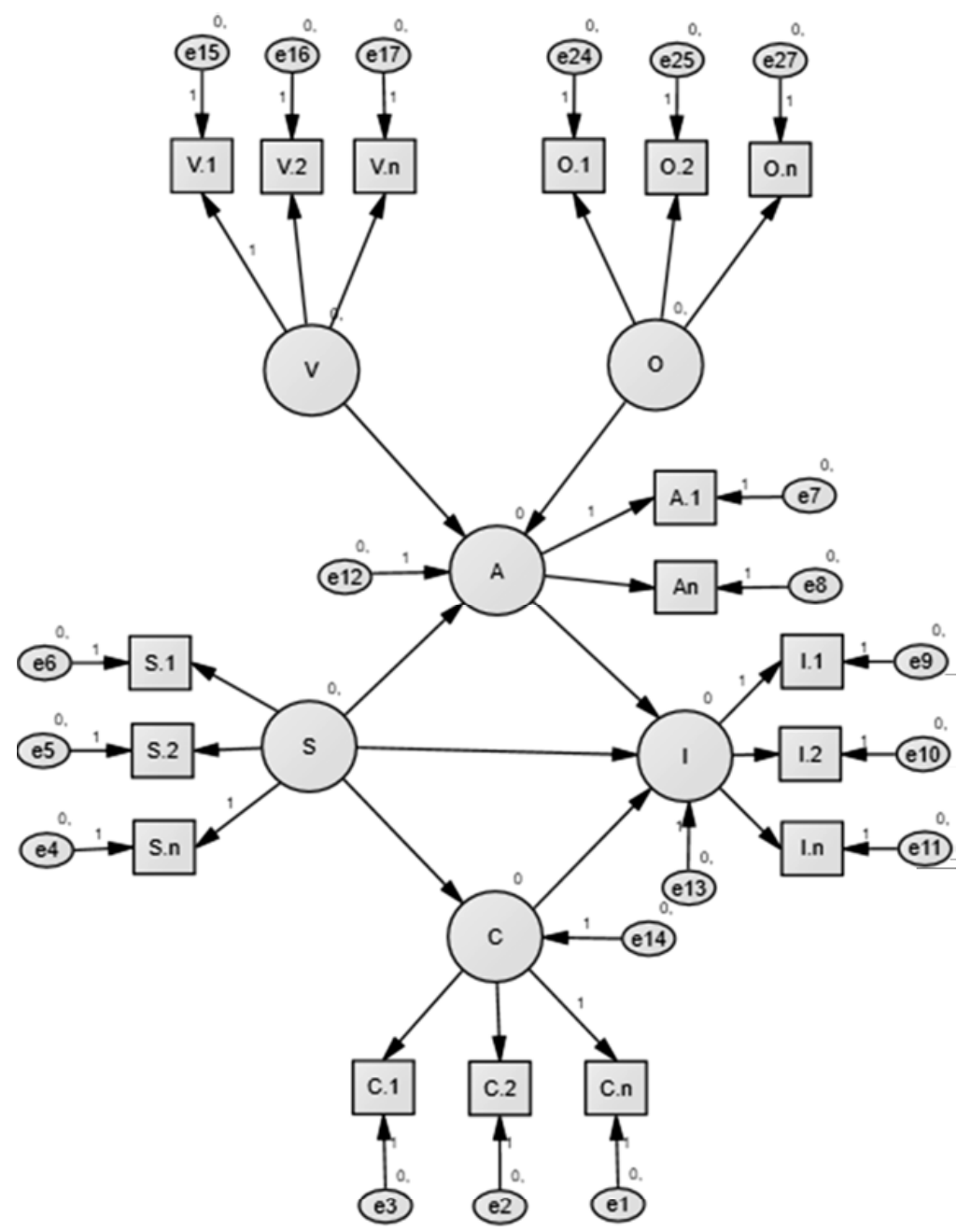

Fuente: Elaboración propia a partir de outputs de AMOS. 
Una vez especificado el modelo de medición se procede a verificar la fiabilidad y la validez de las escalas de medida (paso II), entendidas como el conjunto de ítems utilizados para cada constructo o variables latentes incluido en el modelo teórico, para lo cual se realiza un análisis factorial confirmatorio (AFC).

Para determinar la fiabilidad y la validez del instrumento se calculan los coeficientes de Alfa de Cronbach y de Kaiser Meyer Olkin (KMO) para cada uno de los parámetros (intención emprendedora, actitud de los estudiantes, factor social que los estudiantes tienen, ventajas para emprender, obstáculos para emprender, control de la conducta).

El grado de variabilidad dentro de cada ítem debe ser bajo para considerar que la escala utilizada es fiable. El criterio Alfa de Cronbach permite determinar la consistencia interna de cada constructo. En la tabla 2 puede confirmarse la fiabilidad de las escalas utilizadas para medir los parámetros del modelo, observando que el Alfa Cronbach es superior a 0.7 para la intención emprendedora, las ventajas para emprender, los obstáculos para emprender y la influencia social que los estudiantes tienen, en los que se identifica que existe un incremento al eliminar elementos, sin embargo, no se procede hasta verificar la validez.

Por otro lado, el control de la conducta registra 0.593 y la actitud de los estudiantes 0.253 , lo cual requiere eliminar elementos para alcanzar 0.508; en este caso ninguna de las variables alcanza la fiabilidad deseada, más no se eliminan puesto que su inclusión está fundamentada en la teoría.

Tabla 2. Análisis de fiabilidad y validez.

\begin{tabular}{lcccc}
\multicolumn{1}{c}{ Constructo } & Alfa Cronbach & KMO & Bartlett (sig) & Varianza si elimina $<0.500$ \\
\hline Intención & 0.814 & 0.661 & 0.0000 & 72.902 \\
\hline Actitud & 0.508 & 0.500 & 0.0000 & 69.307 \\
\hline Subjetivo & 0.745 & 0.679 & 0.0000 & 66.241 \\
\hline Control & 0.580 & 0.623 & 0.0000 & 54.786 \\
\hline Ventajas & 0.876 & 0.890 & 0.0000 & 53.852 \\
\hline Obstáculos & 0.864 & 0.898 & 0.0000 & 55.157 \\
\hline
\end{tabular}

Fuente: Elaboración propia a partir del output SPSS.

$\mathrm{El}$ test de esfericidad de Bartlett muestra un nivel de significancia inferior a 0.05 en todos los casos, lo que permite comprobar que los elementos para cada variable no son independientes entre sí y son adecuados para el análisis, es decir, los ítems involucrados para cada variable latente miden un mismo constructo y están fuertemente relacionados.

La medida de adecuación muestral (KMO) se refiere a que el instrumento cumple con los factores teóricos; se encuentra entre 0.5 y 1, por lo que el análisis factorial 
es pertinente para el conjunto de datos debido a que las variables están relacionadas. No obstante, la prueba indica que los elementos con baja comunalidad individual $(<0.55)$, mencionados en la tabla 2 [P8(I), P10(A), P18(C), P19(V), P20(V), P21(V), P22(V), P27(V), P28(V), P32(V), P34(O), P35(O), P36(O), P37(O), P38(O), P39(O), P40(O), P41(O), P42(O), P50(O)], no cumplen con los factores teóricos, por lo que se procedió a eliminarlos para asegurar el ajuste del modelo de ecuaciones estructurales e incrementar la varianza explicada individual de los constructos.

En general, el instrumento cumple con las pruebas de fiabilidad y validez de factores teóricos, aunque resulta necesario señalar que la fiabilidad es cuestionable en la dimensión de control de la conducta y la actitud de los estudiantes, dado que el Alfa de Cronbach arroja un valor menor a 0.7, sin embargo el problema no se solucionó con la eliminación de algún elemento, con ello, a continuación, se procedió al análisis del ajuste del modelo global.

Para la prueba del modelo se realizan pruebas globales de bondad de ajuste o índices de ajuste NFI, CFI, TLI, IFI, Hoelter, PNFI, RMSEA (paso III). Con ello se determinó que el tamaño de muestra es adecuado, puesto que la prueba Hoelter es mayor a 200, asimismo, respecto al CFI o índice de ajuste comparativo de Bentler, se asume que las variables latentes del modelo no se correlacionan entre sí, por lo que se considera aceptable al ser mayor 0.90, al igual que el IFI o índice incremental de ajuste (tabla 3).

Tabla 3. Pruebas globales de bondad de ajuste.

\begin{tabular}{ccccccc} 
CFI & NFI & RMSEA & TLI & IFI & Hoelter $(0.01)$ & PNFI \\
\hline 0.913 & 0.888 & 0.052 & 0.895 & 0.913 & 332 & 0.739 \\
\hline
\end{tabular}

Fuente: Elaboración propia a partir del output SPSS.

Por su parte, como puede observarse, el NFI o índice de ajuste normado de Bentler-Bonett no requiere cumplir los supuestos de Chi-cuadrado. En este caso refleja que el modelo teórico mejora en $88.8 \%$ el ajuste en relación al modelo nulo, muy cercano al mínimo aceptable de 0.90 . Similar a NFI, el TFI está muy cercano al 0.90, lo que permite validar el buen ajuste. Además refleja una buena parsimonia debido a que el PNFI es mayor a 0.60. Por último, se tiene un índice RMSEA satisfactorio al ser menor a 0.08 , y corrige la complejidad del modelo por la discrepancia por grados de libertad.

Con los resultados de estas pruebas se confirma que la especificación del modelo inicial es correcta y adecuada, por lo que no es necesaria la reespecificación del modelo (paso V). 
Una vez que se verificó el ajuste de los datos al modelo teórico se procede a la estimación de parámetros e interpretación de los mismos (pasos IV y VI), probados individualmente con el estadístico t-Student, utilizando el método de máxima verosimilitud (MV); dado que es un análisis de percepción, se consideran problemas de heterogeneidad dependiendo del individuo que responde; este método de estimación permite corregir heterocedasticidad siguiendo el método de mínimos cuadrados ordinarios (MCO), el cual busca maximizar la probabilidad de que las covarianzas observadas sean tomadas de una población.

La figura 3 representa la estimación del modelo, el cual refleja los efectos directos de las variables y los indirectos de una variable latente sobre otra que existe con efecto mediador.

Figura 3. Estimación del modelo.

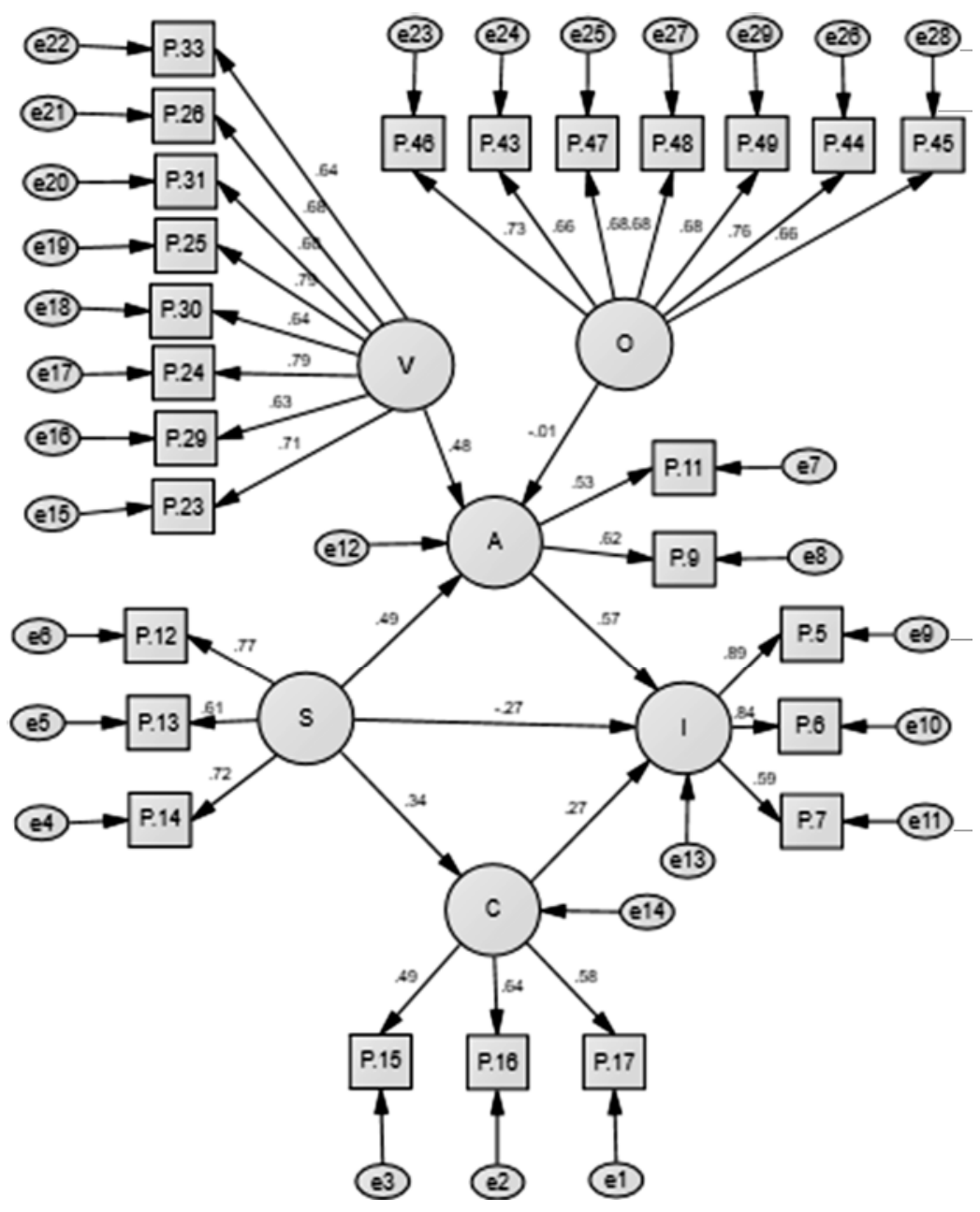

Fuente: Elaboración propia, a partir de los outputs de AMOS. 
Para facilitar la interpretación, en la tabla 4 se enumeran las relaciones que se ejercen entre los parámetros; los coeficientes indican una relación directa y significativa entre los constructos con un soporte del 99\% de confianza, excepto para el modelo 4 , el cual es marginal y no significativo.

Tabla 4. Estimación de parámetros.

\begin{tabular}{|c|c|c|c|c|c|}
\hline \multirow[b]{2}{*}{1} & \multicolumn{3}{|c|}{ Modelo } & \multirow{2}{*}{$\frac{\text { Estimadores (MLE) }}{0.485}$} & \multirow{2}{*}{$\frac{\mathrm{P} \text {-value }}{* * *}$} \\
\hline & Subjetiva & $--->$ & Actitud & & \\
\hline 2 & Subjetiva & $--->$ & Control & 0.343 & $* * *$ \\
\hline 3 & Ventajas & $--->$ & Actitud & 0.481 & $* * *$ \\
\hline 4 & Obstáculos & $--->$ & Actitud & -0.01 & 0.776 \\
\hline 5 & Control & $--->$ & Intención & 0.266 & $* * *$ \\
\hline 6 & Actitud & $--->$ & Intención & 0.573 & $* * *$ \\
\hline 7 & Subjetiva & $--->$ & Intención & -0.271 & $* * *$ \\
\hline
\end{tabular}

Fuente: Elaboración propia a partir del output AMOS.

Los modelos 1, 2 y 7 se corresponden con la variable factor social o norma subjetiva. Estos modelos tienen influencia positiva y directa sobre la actitud (0.485) y el control de la conducta (0.343) de los estudiantes, pero la influencia es negativa sobre la intención emprendedora (-0.271). A su vez, el modelo 5 de control de la conducta ejerce influencia positiva sobre la intención emprendedora (0.266).

Finalmente, la actitud de los estudiantes influye positivamente sobre la intención emprendedora (0.573), como se observa en el modelo 6. En el modelo 3, referente a las ventajas para emprender, se observa influencia positiva y directa sobre la actitud de los estudiantes (0.481), sin embargo, los obstáculos para emprender no respaldan la existencia de influencia sobre la actitud de los estudiantes (-0.01).

Por otra parte, en la tabla 5 se presentan las relaciones para la variable intención emprendedora medida por la actitud de los estudiantes; en el caso de las ventajas y los obstáculos del emprendimiento, para las ventajas tiene influencia positiva indirecta (0.28) y para los obstáculos ejerce efecto negativo (-0.01).

Con respecto al factor social (subjetivo), no solo ejerce un efecto directo sobre la intención emprendedora (-0.27), sino que además genera un efecto indirecto y positivo mediado por la actitud y el control (0.369) (figura 3). Cabe destacar que el efecto total de la variable actitud incide positivamente en mayor medida sobre la intención emprendedora. 
Tabla 5. Efecto indirecto y total.

\begin{tabular}{|c|c|c|c|c|}
\hline & Modelo & & Efecto indirecto & Efecto total \\
\hline Ventajas & $--->$ & & 0.276 & 0.276 \\
\hline Obstáculos & $--->$ & Intención & -0.006 & -0.006 \\
\hline Subjetiva & $-->$ & & 0.369 & 0.098 \\
\hline
\end{tabular}

Fuente: Elaboración propia a partir del output AMOS.

El último aspecto a analizar es la identificación de las posibles diferencias de los estudiantes ante la intención emprendedora entre los dos centros universitarios (CUCEA y CUCEI); se realizó la comparación para diferencia de medias en los factores que explican la intención emprendedora, para ello se generaron las variables promedio para cada una de las variables: intención emprendedora, actitud emprendedora, factor social, control de la actitud, ventajas y obstáculos para emprender, tomando los ítems del cuestionario ajustado según el AFC.

Tabla 6. Diferencia de medias.

\begin{tabular}{|c|c|c|c|c|}
\hline Variable & $\begin{array}{l}\text { CUCEA } \\
\mathrm{n}=751\end{array}$ & $\begin{array}{l}\text { CUCEI } \\
\mathrm{n}=366\end{array}$ & $\begin{array}{c}\text { Prueba Levene } \\
\text { (sig.) }\end{array}$ & $\begin{array}{c}\text { Prueba t Student } \\
\text { (p-value })\end{array}$ \\
\hline Intención & 4.0697 & 3.8834 & 0.000 & 0.001 \\
\hline Actitud & 0.9387 & 0.9286 & 0.160 & 0.425 \\
\hline Ventajas & 4.2557 & 4.1934 & 0.019 & 0.156 \\
\hline Obstáculos & 3.5960 & 3.2314 & 0.986 & 0.000 \\
\hline Control & 0.7182 & 0.6483 & 0.717 & 0.001 \\
\hline Subjetiva & 0.9458 & 0.9396 & 0.387 & 0.600 \\
\hline
\end{tabular}

Fuente: Elaboración propia a partir del output SPSS.

En la tabla 6 los resultados muestran que existen diferencias estadísticamente significativas que nos permiten rechazar la hipótesis nula en las variables intención de emprendimiento, percepción de obstáculos para emprender y control de la conducta, con un $(\mathrm{P}<0.05)$, lo cual indica que existen diferencias entre los dos centros universitarios, comprobando que los promedios para el CUCEA son mayores que para el CUCEI. Esto demuestra que los alumnos del CUCEA opinan que los obstáculos son un impedimento para emprender, lo que resulta razonable, sin embargo la intención la tienen y es factible que se concrete la acción, dado que los aspectos de control están ligados a cómo el estudiante se percibe para convertirse en empresario. 


\section{Discusión}

A la vista de los resultados presentados se puntualizan los hallazgos más relevantes y se comparan con los resultados obtenidos por otros autores, especialmente con los resultados de Valencia, Restrepo y Restrepo (2015) en su análisis cualitativo sobre factores que inciden en la intención emprendedora; Rueda, Fernández y Herrero (2013) con la aplicación de la TAR al ámbito emprendedor en un contexto universitario; Salem (2014), que aplica el modelo de comportamiento planificado (TPB) a una muestra de 120 estudiantes de licenciatura midiendo el impacto al implementar una asignatura de emprendimiento, y Liñán y Chen (2009), que prueban el modelo de intención empresarial en una muestra de 519 individuos con resultados satisfactorios.

La influencia de las variables explicativas sobre la intención emprendedora se refleja como una relación directa y significativa. En general, estos resultados están en la línea de Marulanda, Correa y Mejía (2009), González et al. (2017), Rueda, Fernández y Herrero (2013) y Valencia, Restrepo y Restrepo (2015), que observan una influencia importante de la norma subjetiva, debido a que el soporte familiar es fundamental para el apoyo moral y es fuente de seguridad y confianza para la toma de decisiones, además, conforme los estudiantes perciben la aprobación de su entorno, su actitud y su comportamiento tienden hacia el emprendimiento y, en consecuencia, influye positivamente sobre la intención. En este sentido se manifiestan Skaperdas (1992) y López y Serrano (2017) al considerar la determinación endógena de las preferencias como factor determinante de la asunción de riesgos.

Sin embargo, para Salem (2014), no existe relación directa con la norma subjetiva y la intención emprendedora. En este punto coincide con nuestro estudio, en que de igual forma la norma subjetiva solo tiene efecto a través de las mediadoras que son actitud y control de la conducta. Es posible identificar diferencias en las presiones sociales que modifican la actitud, de acuerdo al entorno cultural, como lo muestra Taiwán con efecto más bajo que España en el estudio de Liñán y Chen (2009), sus resultados indican que las percepciones relevantes se derivan de cómo los valores culturales modifican la forma en que los individuos en cada sociedad perciben el espíritu empresarial.

En este estudio que se presenta se observa que la actitud de los estudiantes influye positivamente en la intención emprendedora, además la relación es más fuerte que el resto de las variables. Un aspecto que cabe resaltar es que la actitud está influenciada por las ventajas y los obstáculos.

Sobre las ventajas para emprender se resalta que tienen efecto directo y positivo; de entre ellas, los estudiantes entrevistados destacaron como principales la independencia económica y en el trabajo, además de la generación de su propio empleo. En este aspecto, los hallazgos de los estudios mencionados al inicio de esta sección destacan la relevancia en los mismos factores sobre su influencia en la actitud emprendedora. 
Con respecto a los obstáculos para emprender sobre la actitud se destaca que la relación es marginal no significativa, por lo que solo será válido si es analizado en esta muestra con un efecto negativo, lo que nos lleva a validar que la actitud emprendedora del estudiante universitario está influenciada negativamente por los obstáculos para emprender, sobresaliendo, entre otros, tener que trabajar demasiadas horas, así como el temor al fracaso y sus consecuencias. Acorde con Valencia, Restrepo y Restrepo (2015) y Rueda, Fernández y Herrero (2013), los obstáculos no son significativos, sin embargo, se puede confirmar que los factores que destacan en este rubro son las horas trabajadas y las consecuencias del fracaso; se infiere la preferencia por emplearse por la seguridad que esto les proporciona, así como menor responsabilidad.

El factor social, que corresponde a la influencia que ejercen los individuos o grupos sociales sobre el estudiante, presenta un efecto directo negativo a la intención emprendedora, sin embargo son positivos indirectamente mediados por el control y la actitud, lo que se suma a cualquier efecto directo el efecto indirecto, como lo validan Liñán y Chen (2009).

Respecto a la variable control de la conducta, se observa influencia positiva sobre la intención emprendedora. Destaca que los estudiantes opinen que si tratasen de crear una empresa tendrían una alta posibilidad de lograrlo. En los estudios comparados se reconoce que esta variable tiene una influencia positiva sobre la intención.

Por último, de las respuestas de los centros universitarios analizados se deriva que existen diferencias entre los estudiantes que estudian ciencias exactas y ciencias económico-administrativas, estos últimos reflejan mayor intención de emprendimiento y control de la conducta, sin embargo tienen mayor percepción de los obstáculos. Este resultado coincide con los estudios de Gasse (1985) y Hisrich (1990), que indicaron que determinados tipos de estudios, como los relacionados con el campo de la economía y la administración de empresas, ayudan especialmente en el proceso emprendedor.

\section{Conclusiones}

De acuerdo con el objetivo establecido para este trabajo y sustentado con la Teoría de Acción Razonada, la evidencia empírica permite concluir que la intención emprendedora de los estudiantes universitarios analizados está influenciada directamente por la actitud, el control de la conducta e indirectamente por los aspectos sociales o norma subjetiva.

Es por tanto que el emprendimiento de los futuros egresados está basado en la creencia, la conveniencia y el impacto personal de los resultados de su conducta; esta afirmación permite validar la hipótesis 1, considerando también cómo se perciben a sí mismos sobre la facilidad de sus intenciones de emprender, hipótesis 2. Con respecto a cómo el alumno percibe los valores, normas o creencia de aquellos a los que respeta y considera referencia que influyen sobre su conducta, hipótesis 3 , 
su influencia directa es negativa, sin embargo, es positiva, mediada por la actitud del estudiante ante la intención de emprender.

Dicho lo anterior podemos concluir que, de acuerdo a la Teoría de Acción Razonada, puesto que la ejecución de acciones puede ser efectiva ante una percepción positiva del control de la conducta y la actitud, dada su racionalidad, de igual forma la presión social de las personas de referencia sobre el estudiante afectará de forma negativa y directa a la intención de emprendimiento; se evidencia también que, si existe una aprobación social, la actitud tendrá mayor efecto sobre la intención. Dicho de otro modo, la norma subjetiva tendrá influencia en la intención solo si el alumno tiene actitud de emprender.

Aunado a lo anterior y conforme a la revisión teórica, la actitud se ve influenciada por las ventajas, hipótesis 4, y los obstáculos percibidos, hipótesis 5, ante la creación de una empresa, donde los factores internos de personalidad y motivación de logro, entendidos como ventajas, potencializan la actitud y, por tanto, la intención de emprender. Por otro lado, los incentivos del gobierno, la viabilidad externa o los inconvenientes obstaculizan la capacidad del individuo, lo que afecta negativamente a la actitud del estudiante ante el emprendimiento y, por consiguiente, a la intención.

Con ello se deja abierto el análisis sobre líneas de actuación pública en los distintos niveles del sistema educativo, una vez que se ha contribuido en el conocimiento sobre los factores y características de los potenciales emprendedores en el ámbito universitario.

Finalmente, hipótesis 6, la evidencia confirma que la opinión del alumno es diferente entre los dos centros universitarios en aspectos relacionados con la intención y el control de la conducta; tiene mayor fuerza en los estudiantes de ciencias económico administrativas, sin embargo también dan mayor peso a los obstáculos, por lo que, aunque existe la intención y por tanto la posibilidad de llevar a cabo la acción, el aspecto de control define la percepción del individuo hacia la decisión de emprender. Por otra parte, los estudiantes de ciencias exactas le dan menos importancia a los obstáculos, lo que nos lleva a suponer que tienen mejores herramientas para enfrentarlos. Esto nos plantea que, para profundizar en el conocimiento de las percepciones empresariales de las distintas formaciones profesionales podría aplicarse el análisis separando los alumnos por cada centro universitario.

\section{REFERENCIAS}

Ackermann, S., y Audretsch, D. B. (eds.) (2013). The economics of small firms: A European challenge (vol. 11). Berlín: Springer Science \& Business Media.

Acs, Z., Audretsch, D., Braunerhjelm, P., y Carlsson, B. (2003). The missing link: The knowledge filter and endogenous growth. DRUID Summer Conference. Copenhague, 12-14 junio.

Ajzen, I., y Fishbein, M. (1974). Factors influencing intentions and the intention-behavior relation. Human Relations, 27(1), 1-15. 
Ajzen, I., y Fishbein, M. (1980). Understanding attitudes and predicting social behavior. Prentice-Hall.

Arroyo, M. (2016). Emprendimiento y universidad emprendedora: conceptualización, propuesta metodológica y caracterización de la Universidad Politécnica de Valencia [Tesis doctoral]. Universidad Politécnica de Valencia, Valencia, España. Recuperado de: http://hdl.handle. net/10261/132663.

Atherton, A., Faria, J. R., Wu, D., y Wu, Z. (2015). Human capital, entrepreneurial entry and survival. Discussion paper in Economics, (1). Nottingham Trent University.

Audretsch, D. B., Keilbach, M., y Lehmann, E. (2005). The knowledge spillover theory of entrepreneurship and technological diffusion. En D. L. Gary (ed.), University entrepreneurship and technology transfer (advances in the study of entrepreneurship, innovation \& economic growth, (vol. 16), (pp. 69-91). Emerald Group Publishing Limited. Recuperado de: doi/10.1016/ S1048-4736(2005)16.

Barrio, S., y Luque, T. (2000). Análisis de ecuaciones estructurales. En L. Teodoro (ed.), Técnicas de análisis de datos en investigación de mercados (pp. 489-557). Madrid: Ediciones Pirámide.

Barsky, R. B., Juster, F. T., Kimball, M. S., y Shapiro, M. D. (1997). Preference parameters and behavioral heterogeneity: An experimental approach in the health and retirement study. The Quarterly Journal of Economics, 112(2), 537-579.

Becker, G. S. (1976). The economic approach to human behavior. University of Chicago Press.

Blázquez, A., Zaldívar, C., y Leite, E. (2018). El emprendimiento, cactitud o como competencia del capital humano? Una mirada conceptual desde la Universidad de Olguín, Cuba. Holos, 8(34), 109-137.

Bollen, K. A. (1989). Structural equations with latent variables. Nueva York: Wiley.

Boyd, N. G., y Vozikis, G. S. (1994). The influence of self-efficacy on the development of entrepreneurial intentions and actions. Entrepreneurship Theory and Practice, 18(4), 63-77.

Breznitz, S. M., y Etzkowitz, H. (2016). University technology transfer: the globalization of academic innovation. Nueva York: Routledge.

Brockhaus, R. H. (1982). The psychology of the entrepreneur. En C. Kent, D. Sexton, y K. Vesper (eds.),
The Encyclopaedia of Entrepreneurship (pp. 39-56). Englewood Cliffs, NJ: Prentice Hall.

Brunello, G. (2002). Absolute risk aversion and the returns to education. Economics of Education Review, 21(6), 635-640.

Clark, B. R. (1998a). The entrepreneurial university: Demand and response. Tertiary Education and $M a-$ nagement, 4(1), 5-16.

Clark, B. R. (1998b). The bigher education system: Academic organization in cross-national perspective. Los Angeles, California, USA: University of California Press.

Cooper, A. C., Woo, C. Y., y Gimeno, F. J. (1994). Initial human and financial capital as predictors of new venture performance. Journal of Business Venturing, 9(5), 351-395.

Diamantopoulos, A. (1994). Modelling with LisRel: A guide for the uninitiated. Journal of Marketing Management, 10(1-3), 105-136.

Echeverri, L., Valencia, A., Benjumea, M., y Barrera, A. (2018). Factores que inciden en la intención emprendedora del estudiantado universitario: un análisis cualitativo. Educare, 22(2), 1-19.

Etzkowitz, H. (2003). Innovation in innovation: The Triple Helix of university-industry-government relations. Social Science Information, 42(3), 293-337.

Etzkowitz, H., y Leydesdorff, L. (2000). The dynamics of innovation: From National Systems and "Mode 2 " to a Triple Helix of university-industry-government relations. Research Policy, 29, 109-123.

Gartner, W. B. (1985). A conceptual framework for describing the phenomenon of new venture creation. Academy of Management Review, 10(4), 696-706.

Gasse, P. (1985). A strategy for the promotion and identification of potential entrepreneurs at the secondary school level. En Frlontiers of Entrepreneurship Research (pp. 538-559). Babson College, Wesllesley, MA.

Godin, B., y Gingras, Y. (2000). The place of universities in the system of knowledge production. Research Policy, 29, 273-278.

González, G., Estrada, C., Leite, E., y Álvarez, J. (2017). Aportaciones al diseño de formación de emprendedores desde la medición de actitudes de estudiantes universitarios. Caso UAEMEX-UPTC (2016). Holos, 2(33), 290-300. 
Guerrero, M., Cunningham, J. A., y Urbano, D. (2015). Economic impact of entrepreneurial universities' activities: An exploratory study of the United Kingdom. Research Policy, 44(3), 748-764. Doi: 10.1016/j. respol.2014.10.008.

Gutiérrez, J. A., Asprilla, E., y Gutiérrez, J. M. (2014). Emprendimiento e investigación en la escala de la formación profesional y la innovación empresarial en Colombia. Revista Escuela de Administración de Negocios, 76, 144-157.

Hirshleifer, J. (1977). Economics from a biological viewpoint. The Journal of Law \& Economics, 20(1), 1-52.

Hisrich, R. D. (1990). Entrepreneurship/intrapreneurship. American Psychologist, 45(2), 209-222.

Jackson, D. L. (2003). Revisiting sample size and number of parameter estimates: Some support for the N: q hypothesis. Structural Equation. Modeling, 10, 128-141.

Kerlinger, F., y Lee, H. (2002). Investigación del comportamiento. Métodos de investigación en las ciencias sociales. México: McGraw-Hill.

Kirby, D. A. (2006). Creating entrepreneurial universities in the UK: Applying entrepreneurship theory to practice. The Journal of Technology Transfer, 31, 599-603.

Kline, R. B. (2005). Principles and practice of structural equation modeling (2a. ed.). Nueva York: Guilford.

Krueger, N. F., Reilly, M. D., y Carsrud, A. L. (2000). Competing models of entrepreneurial intentions. Journal of Business Venturing, 15(5), 411-432.

Liñán, F., y Chen, Y.-W. (2009). Development and cross-cultural application of a specific instrument to measure entrepreneurial intentions. Entrepreneurship: Theory and Practice, 33(3), 593-617.

López, C., y Serrano, A. (2017). Hacia el surgimiento de una cultura de la innovación en un centro público de investigación en México: Hallazgos y aprendizajes. Nova Scientia, 18(9), 313- 341.

Marulanda, F. Á., Montoya, I. A., y Vélez, J. M. (2014). Aportes teóricos y empíricos al estudio del emprendedor. Cuadernos de Administración: Universidad del Valle, 30(51), 90-99. Recuperado de: http://www.scielo.org. $\mathrm{co} / \mathrm{pdf} / \mathrm{cuadm} / \mathrm{v} 30 \mathrm{n} 51 / \mathrm{v} 30 \mathrm{n} 51 \mathrm{a} 10 . \mathrm{pdf}$.

Marulanda, J. A., Correa, G., y Mejía, L. F. (2009). Emprendimiento: visiones desde las teorías del comportamiento humano. Revista EAN, 66, 153-168.
McClelland, D. C. (1961). The achieving society. Nueva York: Van Nostrand. Recuperado de: doi:http://dx.doi. org/10.1037/14359-000.

Nabi, G., Liñan, F., Krueger, N., Fayolle, A., y Walmsley, A. (2016). The impact of entrepreneurship education in higher education: A systematic review and research agenda. Academy of Management Learning and Education, AMLE-2015.

Reynolds, P., Hay, M., y Camp, R. (1999). Global Entrepreneurship Monitor 1999 Report. Londres: London School Business: Babson College, Kauffman Center For Entrepreneurial Leadership.

Ritsila, J., y Tervo, H. (2002). Effects of unemployment on new firm formation: Micro-level panel data evidence from Finland. Small Business Economics, 19, 31-40.

Robson, A. J. (1996). The evolution of attitudes to risk: Lottery tickets and relative wealth. Games and Economic Behavior, 14(2), 190-207.

Rubin, P. H., y Paul, C. W. (1979). An evolutionary model of taste for risk. Economic Inquiry, 17, 585-596. Recuperado de: doi:10.1111/j.1465-7295.1979.tb00549.x.

Rueda, S. I., Fernández, L. A., y Herrero, C. Á. (2013). Aplicación de la teoría de la acción razonada al ámbito emprendedor en un contexto universitario. Investigaciones Regionales, 26, 141-158.

Salem, O. M. (2014). Variables explicativas de la intención emprendedora de los estudiantes universitarios y la importancia de la materia emprendimiento como un factor. Compendium: Cuadernos de Economía y Administración, 1(1).

Sampat, B. N., Mowery, D. C., y Ziedonis, A. A. (2003). Changes in university patent quality after the BayhDole act: A re-examination. International Journal of Industrial Organization, 21(9), 1371-1390.

Schumpeter, J. (1950). Capitalism, socialism and democracy (3a. ed.). Nueva York: Harper.

Shapero, A. (1975). The displaced, uncomfortable entrepreneur. Psychology Today, 6(9), 83-88.

Shapero, A., y Sokol, L. (1982). Social dimensions of entrepreneurship. En C. Kent, D. Sexton, y K. Vesper (eds.), Encyplopedia of entrepreneurship (pp. 72-90). Englewood Cliffs, NJ: Prentice Hall.

Skaperdas, S. (1992). Cooperation, conflict, and power in the absence of property rights. The American Economic Review, 82(4), 720-739. 
Thurik, A. R., Stam, E., y Audretsch, D. B. (2013). The rise of the entrepreneurial economy and the future of dynamic capitalism. Technovation, 33(8), 302-310.

Valencia, A., Restrepo, M. I., y Restrepo, M. A. (2015). Factores explicativos de las intenciones emprendedoras en estudiantes universitarios. Espacios, 36, 05. Van de Ven, A. H., Hudson, R., y Schroeder, D. M. (1984). Designing new business startups: Entrepre- neurial, organizational, and ecological considerations. Journal of Management, 10(1), 87-107.

Waldman, D. A. (1994). The contributions of total quality management to a theory of work performance. Academy of Management Review, 19(3), 510-536.

Xerfan, C., Ribeiro, A., y Nunes, S. (2018). El diseño como modelo de formación emprendedora en educación universitaria. Centro de estudios en diseño y comunicación, 263-278.

\section{Anexo}

\section{Cuestionario}

I. Datos generales

\begin{tabular}{|c|c|c|c|}
\hline & P.1 & Sexo & $\begin{array}{l}1 \text { Hombre } \\
0 \text { Mujer }\end{array}$ \\
\hline & P.2 & Edad & \\
\hline & P.4 & ¿Cuál carrera estás cursando? & \\
\hline $\begin{array}{l}\text { Intención (I) } \\
\end{array}$ & P.5 & ¿Has pensado seriamente en crear una empresa o negocio propio? & $1 \mathrm{Nada}$ \\
\hline & P.6 & ¿Estas decidido a crear tu propia empresa en el futuro? & 2 Poco \\
\hline & P.7 & ¿Consideras que crear tu propia empresa es una buena opción de autoempleo? & 3 Medianamente \\
\hline & P.8 & $¿$ Crees que tener estudios de formación profesional es importante para & 4 Altamente \\
\hline & & garantizar el éxito de una empresa propia? & 5 Totalmente \\
\hline$\overline{\text { Actitud (A) }}$ & P.9 & Si tuvieras oportunidad y recursos ¿te gustaría crear una empresa? & $1 \mathrm{Nada}$ \\
\hline & P.10 & Entre varias opciones ¿preferirías emprender tu propio negocio? & 2 Poco \\
\hline & P.11 & Ser un empresario ¿te supondría una satisfacción? & 3 Medianamente \\
\hline & & & 4 Altamente \\
\hline & & & 5 Totalmente \\
\hline Aspectos & P.12 & Mis amigos aprobarían mi decisión & \\
\hline subjetivos $(\mathrm{S})$ & P.13 & Mi familia más directa aprobaría mi decisión & \\
\hline & P.14 & Mis compañeros aprobarían mi decisión & \\
\hline Aspectos de & P.15 & Crear una empresa y mantenerla en funcionamiento sería fácil para mí & \\
\hline control (C) & P.16 & Puedo mantener bajo control el proceso de creación de una empresa & \\
\hline & P.17 & Si tratase de crear una empresa, tendría una alta probabilidad de lograrlo & \\
\hline & P.18 & Conozco los detalles prácticos necesarios para crear una empresa & \\
\hline Ventajas (V) & P.19 & Poner en marcha ideas propias & 1 Totalmente \\
\hline & P.20 & Aprovechar los conocimientos adquiridos en la universidad & en desacuerdo \\
\hline & P.21 & Poner a prueba tus habilidades y destrezas & 2 En desacuerdo \\
\hline & P.22 & Crear y ofrecer algo nuevo al mercado & 3 Medianamente \\
\hline & P.23 & Generar tu propio empleo & de acuerdo \\
\hline & P.24 & Ser independiente económicamente & 4 De acuerdo \\
\hline & P.25 & Ser independiente en el trabajo & 5 Totalmente \\
\hline & P.26 & Incrementar los ingresos & de acuerdo \\
\hline & P.27 & Seguir la tradición familiar & \\
\hline & P. 28 & Poder ayudar a tus familiares & \\
\hline & P.29 & Tener control del tiempo propio & \\
\hline
\end{tabular}




\begin{tabular}{|c|c|c|c|}
\hline \multirow[t]{5}{*}{ Ventajas (V) } & P.30 & Ser tu propio jefe & 1 Totalmente en desacuerdo \\
\hline & P.31 & Lograr desarrollo y realización personal & 2 En desacuerdo \\
\hline & P.32 & Dirigir un grupo de personas & 3 Medianamente de acuerdo \\
\hline & P.33 & Invertir y conseguir un patrimonio personal & 4 De acuerdo \\
\hline & P.33 & Invertir y conseguir un patrimonio personal & 5 Totalmente de acuerdo \\
\hline \multirow[t]{17}{*}{ Obstáculos (O) } & P.34 & Desconocimiento de la inversión necesaria para iniciar la actividad & \\
\hline & P.35 & Falta de capital para iniciar & \\
\hline & P.36 & Dificultades para conseguir créditos & \\
\hline & P.37 & Falta de experiencia en el tipo de negocio a iniciar & \\
\hline & P.38 & Desconocimiento técnico del tipo de negocio que se va a iniciar & \\
\hline & P.39 & Cargas fiscales excesivas & \\
\hline & P.40 & Demasiadas normas y leyes que cumplir se desconocen & \\
\hline & P.41 & Riesgo económico & \\
\hline & P.42 & Dificultad para encontrar personal competente & \\
\hline & P.43 & Probabilidad de fracaso & \\
\hline & P.44 & Responsabilidad demasiado grande para asumirla & \\
\hline & P.45 & Falta de información sobre el mercado & \\
\hline & P.46 & Temor al fracaso y sus consecuencias & \\
\hline & P.47 & Ingresos irregulares y no seguros & \\
\hline & P.48 & Problemas con el personal & \\
\hline & P.49 & Tener que trabajar demasiadas horas & \\
\hline & P. 50 & Mala imagen de los empresarios & \\
\hline
\end{tabular}

Fuente: Elaboración propia.

Cómo citar este artículo:

Contreras Cueva, A. B., González Morales, O. y Macías Álvarez, P. (2020). Intención emprendedora de los estudiantes universitarios. IE Revista de Investigación Educativa de la REDIECH, 11, e-780. doi: 10.33010/ie_rie_rediech.v11i0.780. 